\title{
Retrieval of Consolidated Spatial Memory in the Water Maze Is Correlated with Expression of pCREB and Egr1 in the Hippocampus of Aged Mice
}

\author{
GuoXia Zhou $^{a-c}$ WanXia Xiong ${ }^{a, b}$ XiaoGuang Zhang a, b ShengJin Ge ${ }^{a, b}$

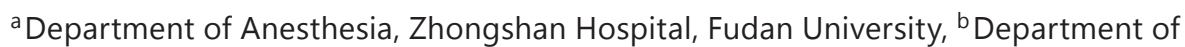 \\ Anesthesiology, Shanghai Medical College, Fudan University, and 'Department of \\ Anesthesia, Shanghai Fifth People's Hospital, Fudan University, Shanghai, China
}

\section{Key Words}

Hippocampus - Cyclic AMP response element-binding protein - Early growth response protein 1 . Spatial probe test . Aged mouse

\begin{abstract}
Objective: To study the relationship of the expression of phosphorylated cyclic AMP response element-binding protein ( $\mathrm{PCREB}$ ) and early growth response protein 1 (Egr1) in the hippocampus of aged mice with retrieval of consolidated spatial memory in a water maze. Methods: Twenty-four aged mice were allocated into no training or probe test (naïve), no training but exposed to the same probe test (NTPRT), received training and probe test (PRT), and received training but no probe test (NPRT) groups. Twelve mice were trained in a water maze over 14 days. After the final probe trial on day 15 , all mice were anesthetized and the brains were removed. pCREB immunoreactivity (pCREB-ir) and Egr1 immunoreactivity (Egr1-ir) in the hippocampal CA1 and CA3 areas were examined. Results: pCREB-ir and Egr1-ir in the CA1 and CA3 areas of the NPRT and PRT groups were significantly higher than those of the naïve and NTPRT groups, and those in the PRT group were significantly higher than in the NPRT group. In all groups, pCREB-ir was significantly higher in the CA3 area compared to the CA1 area, while Egr1-ir was significantly higher in the CA1 area compared to the CA3 area. Conclusion: Retrieval, as well as formation, of consolidated spatial memory in the water maze is correlated with expression of $\mathrm{pCREB}$ and Egr1 in the hippocampus of aged mice.
\end{abstract}

Copyright (C) 2013 S. Karger AG, Basel

GuoXia Zhou and WanXia Xiong contributed equally to this work.

ShengJin Ge

Department of Anesthesia, Zhongshan Hospital

Fudan University, No. 180 Fenglin Road

Shanghai 200032 (China)

E-Mail ge.shengjin@fudan.edu.cn 
Zhou et al.: Retrieval of Consolidated Spatial Memory in the Water Maze Is Correlated with Expression of pCREB and Egr1 in the Hippocampus of Aged Mice

\section{Introduction}

According to their temporal stability, memories can be divided into at least 2 distinct forms: short-term memory (STM) and long-term memory (LTM). Formation of LTM is dependent on gene regulation and de novo protein synthesis that are required for synaptic enhancement [1]. The cyclic AMP response element-binding protein (CREB) is a key transcription factor that has been implicated in LTM formation across different species [2-5]. Phosphorylation/activation of the transcription factor CREB (pCREB) on Ser 133 by cyclic AMP- or $\mathrm{Ca}^{2+}$-dependent protein kinase is critical for LTM consolidation [6-8]. Numerous studies have shown that spatial memory formation was associated with increased pCREB within the hippocampus [9-11], while decreased levels of CREB or disruption of CREBdependent transcription in the dorsal hippocampus interferes with spatial memory without affecting STM [5, 12-14]. The early growth response protein 1 (Egr1), also known as zif268, Krox24, TZs8, Zenk, or NGF-I-A [15], is an immediate early gene encoding the zinc finger transcription factor of the Egr family and is regulated by CREB activity [16].

Previous work has shown that CREB is crucial for the consolidation of long-term conditioned fear memories, but not for encoding, storage, or retrieval of these memories [17]. The present study is aimed to investigate the expression of pCREB and Egr1 in aged mice during spatial probe test to evaluate retrieval of consolidated spatial memory in the Morris water maze.

\section{Animals and Methods}

This study was approved by the Committee on the Care and Use of Laboratory Animals, Zhongshan Hospital, Fudan University, Shanghai, China.

\section{Animals}

A total of 24 male mice of the C57BL/ 6 strain (8 months old at arrival; Shanghai Laboratory Animal Center, Chinese Academy of Sciences) were studied. Mice were housed in groups of 6 to a cage. They were maintained on a 12-hour light-dark artificial cycle (lights on at 7:00 a.m.) in a temperature $\left(22^{\circ} \mathrm{C}\right)$ - and humidity-controlled $(60 \%)$ colony room and had ad libitum access to food and water. The experiment started when the mice were 16 months old and weighing approximately 25-35 g. They were tested during the light phase between 7:00 a.m. and 7:00 p.m.

C57BL/ 6 mice were chosen because they are one of the background strains commonly used to construct transgenic mouse models with the goal of identifying molecular mechanisms critical for learning and memory function. In the strategy preference test, C57BL/6 mice preferred a place strategy and expressed higher levels of pCREB in the hippocampus after place training [18].

\section{Apparatus}

The water maze consisted of a swimming pool based on that described by Morris [19] and adapted for mice. It consisted of a circular tank $(120 \mathrm{~cm}$ in diameter, $50 \mathrm{~cm}$ in height), filled to a depth of $30 \mathrm{~cm}$ with water maintained at $25 \pm 1^{\circ} \mathrm{C}$. We chose a white maze to improve the image quality and avoid the addition of white nontoxic paint or milk which might have had an impact on the results. The pool was located in a room uniformly illuminated by a halogen lamp and equipped with various distal cues. Located inside the pool was a removable, circular (10 cm in diameter) platform made of transparent Plexiglas, positioned such that its top surface was $1.0 \mathrm{~cm}$ below the water surface. The platform, which served as refuge from the water, is generally located in the center of an arbitrarily defined quadrant of the maze. 
The located quadrant platform was defined to be the target quadrant. Data were collected using a video camera fixed to the ceiling of the room and connected to a video recorder and to a video tracking system (Videotrack; Viewpoint).

\section{Behavioral Procedures}

\section{Pretraining}

Three days prior to the acquisition phase, each mouse received a pretraining session that consisted of (1) placing the mouse on the platform where it had to stay for at least $15 \mathrm{~s}$, (2) a 60 -second swimming period, and (3) several trials of climbing onto the platform until each animal was able to climb without help. This nonspatial procedure was required to avoid confusion between procedural aspects of the task and subsequent spatial performance [20].

General Training Procedure

During the place navigation test, which lasted for 14 days, mice were subjected to a daily 4-trial session. Each trial consisted of releasing the mouse into the water facing the outer edge of the pool at one of the quadrants and letting the animal escape to the submerged platform before $60 \mathrm{~s}$ had elapsed. A trial terminated when the animal reached the platform, where it remained for $15 \mathrm{~s}$. Mice that failed to find the platform within this time limit were placed onto the platform by the experimenter and had to stay there for $15 \mathrm{~s}$ before being removed and placed back in their home cage for an equal intertrial interval. The releasing point differed in each trial and different sequences of releasing points were used from day to day. On the 4th, 7th, 11th, and 14th day, the animals underwent probe trials at 2 different releasing points, replacing the 4-trial session, which consisted of letting the mouse swim in the pool while the platform was removed for a fixed duration (60 s) as averaged. Animal movements were recorded using the video track system. The computer data were processed using Excel. This processing allowed us to calculate the escape latency (time required to find the platform, in seconds) and the percentage of time spent in the target quadrant. In percentages, more than $30 \%$ meant the acquisition of the spatial reference memory. On day 14, all 12 aged mice were familiar with the maze and they were randomized into the no probe test (NPRT) group and the probe test (PRT) group. On day 15, the PRT group was subjected to a probe test while the NPRT group was not. Mice of the NTPRT (no training but exposed to the same probe trial experience) group $(\mathrm{n}=6)$ were submitted to a probe test after a 3-day pretraining session and sacrificed together with the PRT and NPRT groups. The undisturbed mice of the naïve group $(n=6)$ were handled similarly to the other trained animals prior to the acquisition phase, and taken out of their home cages to be sacrificed altogether with the PRT, NPRT and NTPRT groups.

All behavioral measures were analyzed on day 14 by a repeated measure analysis of variance (ANOVA) and measures of the NPRT and PRT groups using the independent samples t test (SPSS Statistics 17.0 ), with $\mathrm{p}<0.05$ considered statistically significant.

\section{Immunohistochemical Procedures}

On day 15, mice were deeply anesthetized at 15 min after the probe trial and perfused transcardially with ice-cold phosphate-buffered solution (PBS) and 4\% paraformaldehyde. The brains were removed, postfixed overnight in $4 \%$ paraformaldehyde/PBS, and placed in 30\% sucrose until they sank. Coronal sections $(30 \mu \mathrm{m})$ were cut on a microtome and collected in PBS.

\section{Immunofluorescence}

Blocking of nonspecific epitopes was performed with 3\% serum and 0.1\% bovine serum albumin in PBS with $0.3 \%$ Triton $\mathrm{X}$ for $30 \mathrm{~min}$ at room temperature and then in the primary antibody (Phospho-CREB Rabbit mAb, 1:200 or Egr1 Rabbit mAb, 1:200; Cell Signaling). Sections were incubated for $48 \mathrm{~h}$ at $4{ }^{\circ} \mathrm{C}$ and then washed in PBS and incubated for $2 \mathrm{~h}$ with a 
Zhou et al.: Retrieval of Consolidated Spatial Memory in the Water Maze Is Correlated with Expression of pCREB and Egr1 in the Hippocampus of Aged Mice

1:200 dilution of $\mathrm{Cy} 3$ goat anti-rabbit IgG antisera (Jackson). Nuclear counterstaining was performed with 4',6-diamidino-2-phenylindole (DAPI; Sigma) for $30 \mathrm{~min}$ at room temperature before 3 washes with $\mathrm{dH}_{2} \mathrm{O}$ for 1 min each. Afterwards the sections were coverslipped.

\section{Quantification and Analysis of Immunohistochemical Data}

Quantitative analysis was performed using an imaging analysis system (Leica QWin). The experimenter was blind to the experimental groups of the examined mice. For all analyses of regions, counts were taken bilaterally with a $20 \times$ objective from at least 5 consecutive sections per animal and the grey of positive nuclei per square micrometer was averaged to produce a mean. Quantification of pCREB- and Egr1-immunoreactive neurons was performed in the CA1 and CA3 area of the dorsal hippocampus. Immunohistochemical data were expressed as mean \pm SEM and analyzed by ANOVA (4 groups) and the paired samples t test (CA1 vs. CA3; SPSS Statistics 17.0). Significance of results was accepted at $\mathrm{p}<0.05$.

\section{Results}

\section{Behavioral Results}

The training protocol led to a good consolidated spatial memory of the platform location. Figure 1 shows the performance of the mice from day 1 to day 14 . The mice progressively learned the task, as indicated by decreasing latencies from day 1 to day 13 and increasing percentages from day 4 to day 14 . This was confirmed by analyses of variance performed on data from the whole training period (from day 1 to day 14), yielding the main effect time $\left(F_{9,126}=22.08, p<0.0001\right.$; fig. $\left.1 \mathrm{a}\right)$, and the main effect was on day $12(\mathrm{~F}=152.41, \mathrm{p}<0.0001)$. When analyzing performance from day 4 to day 14, statistical analyses showed significant effect times $\left(F_{3,39}=11.32, p<0.0001\right.$; fig. $\left.1 b\right)$, preceding the asymptotic level of performance reached at day 11 (data not shown). Memory of the platform location was assessed with the probe trial on days $4,7,11$, and 14 . Percentages of the time spent in the target quadrant relative to the opposite quadrant $(t=229.448, p<0.0001$; fig. $1 \mathrm{c})$ revealed a great retention of the platform location. There was no difference in the performance of day 14 between the NPRT and PRT groups $(t=0.075, p=0.789$; fig. $1 d)$. There was no thigmotaxis and floating behavior during training, and statistical analyses of the mean swim speed during training did not reveal any significant group effect $(\mathrm{F}<1, \mathrm{p}>0.3)$.

\section{Immunohistochemical Results}

Can the probe test trigger the expression of pCREB and Egr1 in the hippocampus of mice? To solve this question, we compared the levels of pCREB and Egr1 in the naïve, NTPRT, NPRT and PRT groups. All mice were sacrificed at $15 \mathrm{~min}$ after the final probe trial on day 15 (fig. 2). Activation of the ERK/CREB pathway in the CA1 area was at 0-15 min after paired conditioning, but at $0-1$ and $9-12 \mathrm{~h}$ after unpaired conditioning as revealed by immunocytochemistry and Western blotting. Egr1 protein induction reached its maximum levels after approximately $1-2 \mathrm{~h}$ by various types of stimulation $[21,22]$ and a biphasic pattern of CREB phosphorylation in the hippocampal CA1 area (at $15 \mathrm{~min}$ and $9 \mathrm{~h}$ ), whereas a monophasic pattern was detected in the CA3 area (15 $\mathrm{min})$ after the final probe trial on day 9 [23]. So we selected the time point of $15 \mathrm{~min}$ after the final probe test on day 15 to sacrifice the mice. The results showed that the levels of pCREB immunoreactivity (pCREB-ir; fig. 2a, c) and Egr1 immunoreactivity (Egr1-ir; fig. 2b, c) in the CA1 and CA3 areas of the NPRT group were significantly lower than those of the PRT group (all $p$ values $<0.05$ ). The pCREB-ir and Egr1-ir levels in both the CA1 and CA3 areas of the NPRT and PRT groups were significantly higher than those of the naïve and NTPRT controls (all p values $<0.05$ ), and there was no difference between 


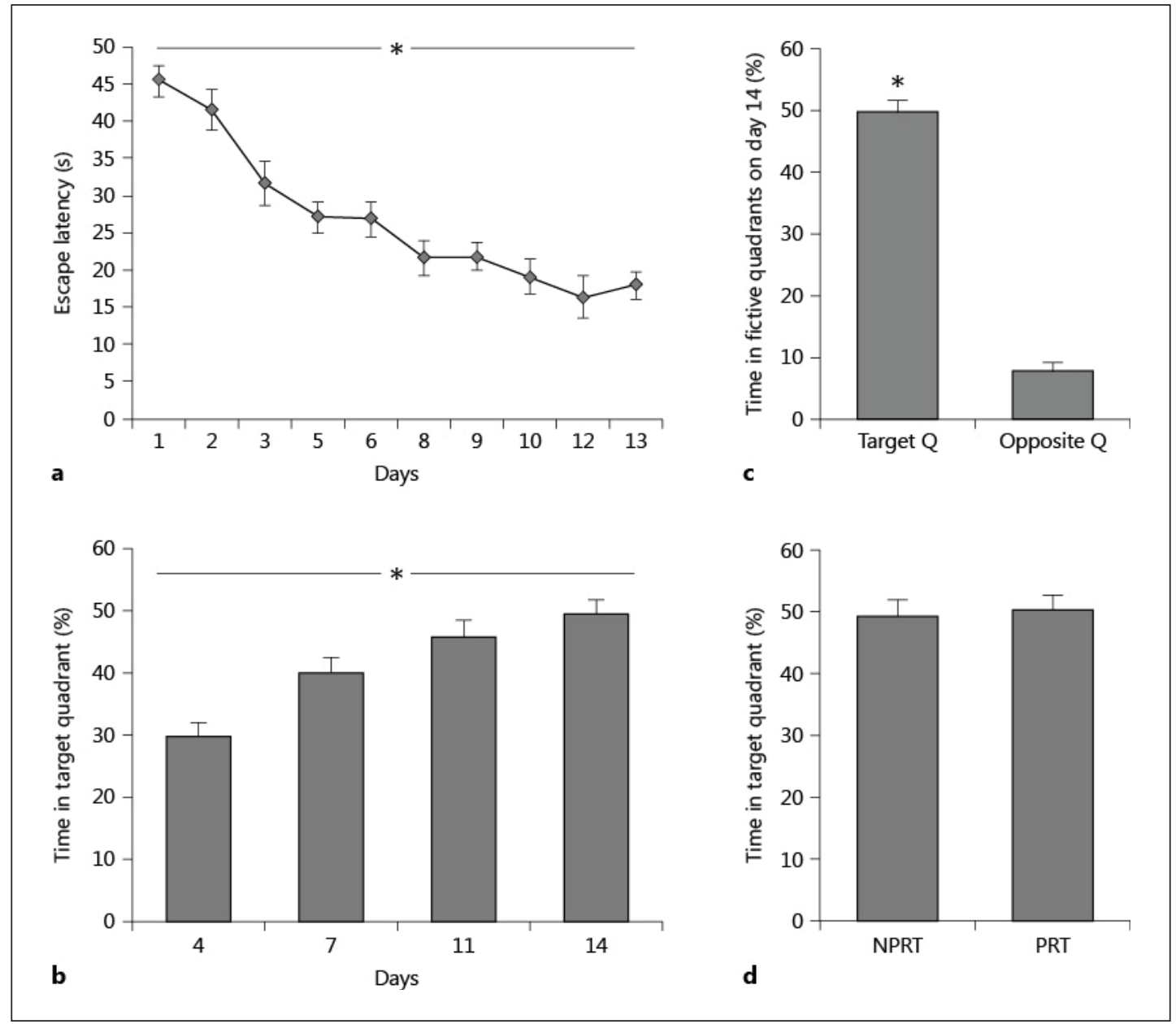

Fig. 1. Acquisition of the spatial reference memory task. a Mice acquired the reference memory task as expressed by the mean escape latency after training sessions. b, c Memory of the platform location was assessed by probe tests on days $4,7,11$, and 14 . The percentages of the time spent in the target quadrant reveal a great retention of the platform location. $\mathbf{d}$ No difference was observed in the percentages of time spent in the target quadrant between the PRT and NPRT groups. Data are mean \pm SEM; $n=6$ for the NPRT or PRT groups. Behavioral measure data of both groups on day 14 were analyzed using the independent samples t test: $p=0.982$, and other data were analyzed using a repeated measure ANOVA: ${ }^{*} p<0.0001$.

naïve and NTPRT groups (all p values >0.05). In all groups, the level of pCREB-ir (fig. 2a, c) was significantly higher in the CA3 area compared to the CA1 area (all p values $<0.05$ ), while the level of Egr1-ir (fig. 2b, c) was significantly higher in the CA1 area compared to the CA3 area (all $\mathrm{p}$ values $<0.05$ ).

\section{Discussion}

The present study demonstrated an increased expression of pCREB and Egr1 in the dorsal hippocampus during spatial probe test to evaluate retrieval of consolidated spatial memory. In this study, all mice in the PRT and NPRT groups acquired spatial memory and did not differ in the acquisition of a platform location in the Morris water maze by the end of 


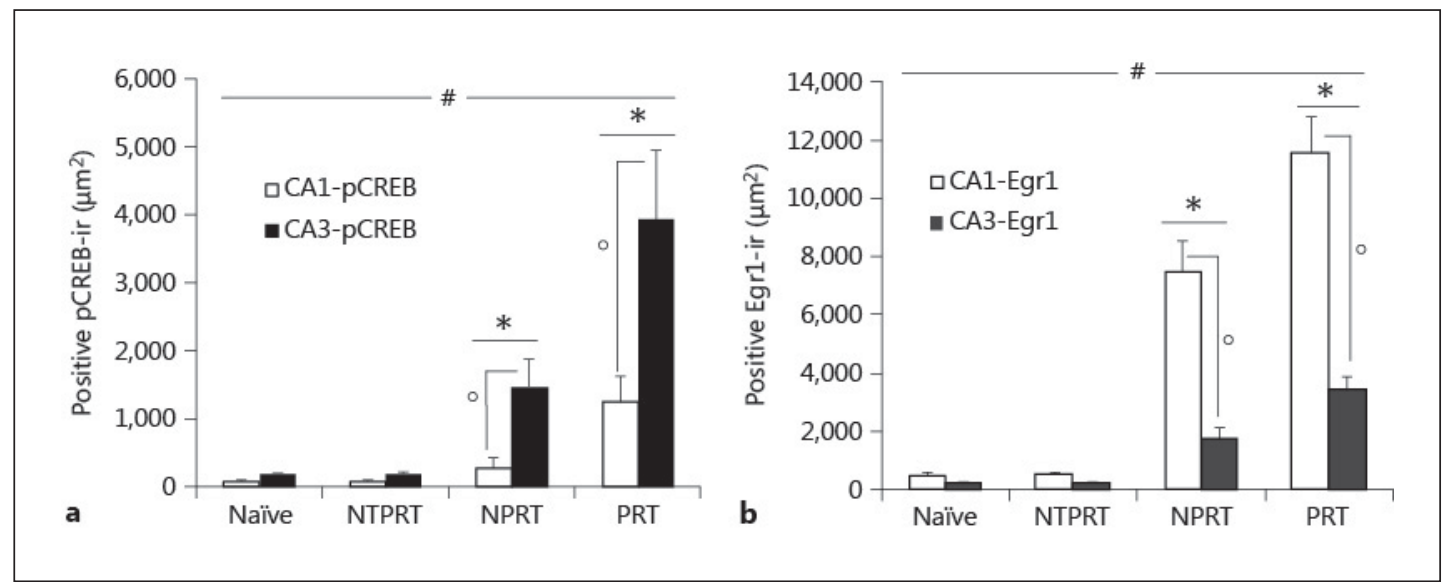

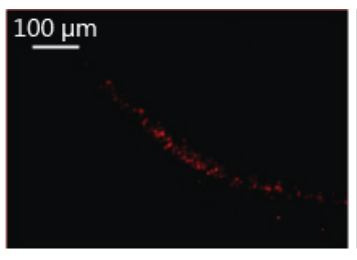

Naïve-Egr1-CA1

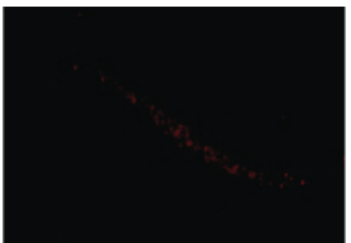

NTPRT-Egr1-CA1

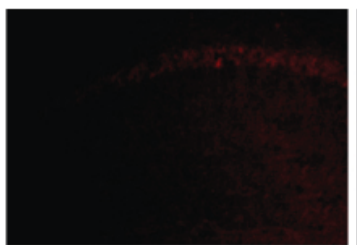

NPRT-Egr1-CA1

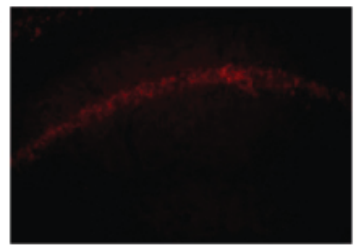

c

PRT-Egr1-CA1

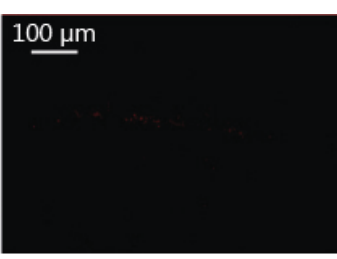

Naïve-pCREB-CA1

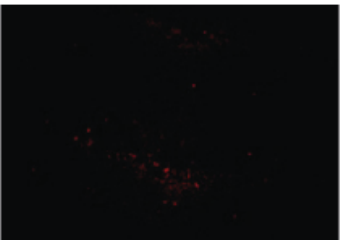

NTPRT-pCREB-CA1

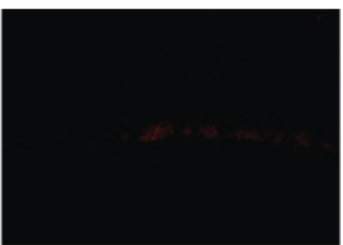

NPRT-pCREB-CA1

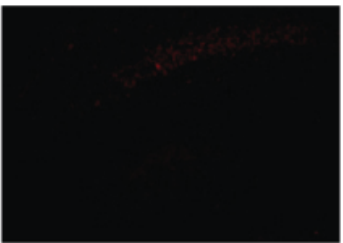

PRT-PCREB-CA1

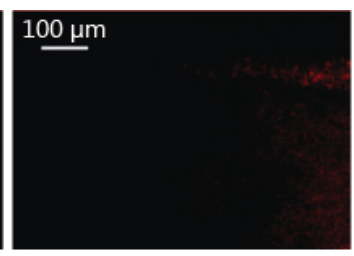

Naïve-Egr1-CA3

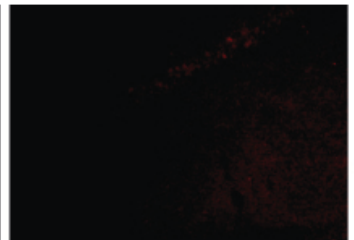

NTPRT-Egr1-CA3

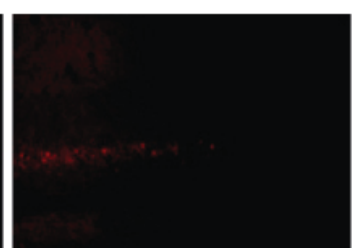

NPRT-Egr1-CA3

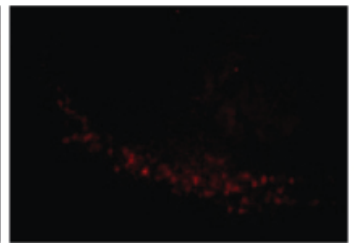

PRT-Egr1-CA3

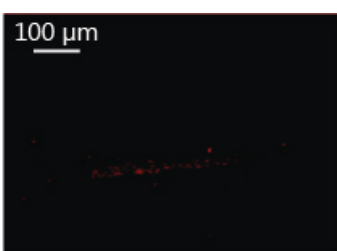

Naïve-pCREB-CA3

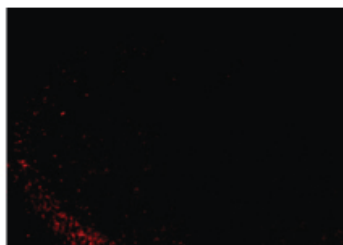

NTPRT-PCREB-CA3

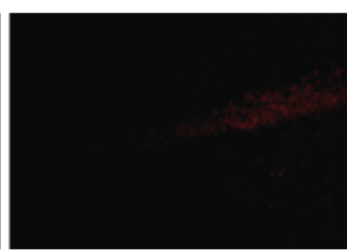

NPRT-pCREB-CA3

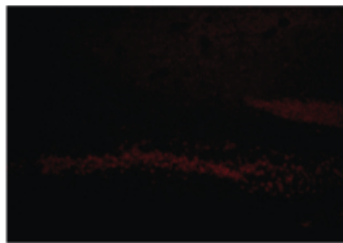

PRT-PCREB-CA3

Fig. 2. The probe tests triggered upregulation of pCREB and Egr1 in the hippocampus of the PRT group. a pCREB-ir levels in the CA1 and CA3 areas of all groups. b Egr1-ir levels in the CA1 and CA3 areas of all groups. c Representative photomicrographs illustrating pCREB-ir and Egr1-ir in the CA1 and CA3 areas of all groups. Data are mean \pm SEM; $n=6$ for each experimental group. Immunohistochemical data were analyzed by ANOVA ( $p>0.05$ : naïve vs. NTPRT; ${ }^{*} p<0.05$ : NPRT and PRT vs. naïve or NTPRT; ${ }^{\circ} \mathrm{p}<0.05$ : NPRT vs. PRT) and by the paired samples test ( ${ }^{\#} \mathrm{p}<0.05$ : CA1 vs. CA3). 


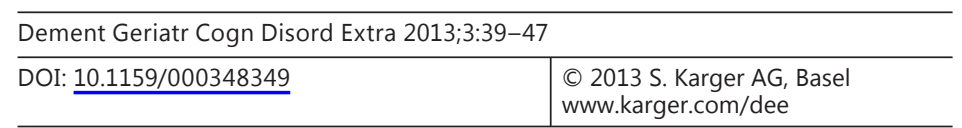

Zhou et al.: Retrieval of Consolidated Spatial Memory in the Water Maze Is Correlated with Expression of pCREB and Egr1 in the Hippocampus of Aged Mice

training. Therefore, we can conclude that in the PRT group, the increase of pCREB and Egr1 in the hippocampus is due to the probe test rather than enhanced learning. However, expression of pCREB and Egr1 in the NTPRT group is significantly lower than in the PRT group. Our findings are supported by a previous study, which found that a strong CA1 CREB phosphorylation was observed immediately after training irrespective of acquisition of the behavior. In contrast, at 15 min after training, the changes in the CA1 CREB phosphorylation state were specifically related to the individual behavioral performance [23].

Considerable studies have reported that acquisition of spatial learning was associated with a progressive increase in PCREB in the hippocampus [11,23-25], and place learning was facilitated by expression of CREB in the dorsal hippocampus [7]. Overexpression of dCREB2- $\alpha$, the Drosophila equivalent of mammalian CREB, enhanced the formation of long-term olfactory learning [26]. Blockade of ERK1/2 activation disrupted retention of unpaired and paired conditioning, but did not affect STM [8]. Since CREB is a downstream target of ERK1/2, inhibition of ERK1/2 activation led to the blockade of CREB activation $[27,28]$. In addition, overexpression of dominant negative CREB in the dorsal hippocampus eliminated spatial bias in the water maze [12], and overexpression of mutant CREB blocked LTM, but not STM, for a socially transmitted food preference [29]. Costa-Mattioli et al. [30] examined the effects of forskolin or 4 trains at $100 \mathrm{~Hz}$ [both induce late long-term potentiation (LTP) and stimulate CREB-mediated gene expression] in wild-type and GCN2-/- slices. Both protocols showed that ATF4 was increased and that CREB and expression of the immediate early gene Egr-1, regulated by CREB, were decreased in the GCN2-/- mice, which was associated with a specific impairment of hippocampus-dependent learning and memory, including contextual fear conditioning and spatial memory tested in the Morris water maze after more intense training. CREB inactivation at approximately $4 \mathrm{~h}$ after training blocked memory storage of different tasks [5, 12] and also LTP late maintenance (i.e. persistence beyond 2-4 h) [31, 32]. The study of Guzowski and McGaugh [5] showed that pretraining intrahippocampal infusion of CREB antisense oligodeoxynucleotides produces disruptions in CREB protein levels, and this disruption impairs retention in rats trained in the Morris water maze, suggesting an importance of CREB in the consolidation of memory processes. The activation of Egr1 is necessary for the generation of late LTP or for the formation of LTM [33]. Genetic studies in mice have also supported the conclusion that Egr1 is critical for memory formation. Egr1 knockout mice show impaired LTM but intact STM, including spatial learning [15].

We used immunohistochemistry to investigate whether probe trials induce expression of pCREB and Egr1 in the dorsal hippocampus (both in the CA1 and CA3 areas). We chose the hippocampus because it has been demonstrated that the CA3 network can support a recall mechanism named pattern completion [34], which could be involved in some instances of contextual fear recall [35]. Our results show that expression of Egr1 is significantly higher in the CA1 area compared to the CA3 area, while pCREB is significantly higher in the CA3 area. An in situ hybridization study [36] demonstrated that basal expression of Egr1, without intentional neuronal stimulation, is strongest in the CA1 area but very low in the dentate gyrus. Our findings are consistent with the view that has emerged from a previous study in contextual fear memory, which reports that contextual information is rapidly processed in the autoassociative CA3 network and sent to the CA1 network to be stored ultimately in the neocortex [37]. Our results are also strengthened by previous work showing that CA3 NMDA receptors are crucial for rapid hippocampal encoding of unique events [38]. The levels and activities of CREB in the neuron might differ dramatically during acquisition and retrieval. The current results show that the expression of pCREB and Egr1 increases in the hippocampus during probe test after retrieval of consolidated spatial memory. Several studies have shown that the expression of Egr1 increases in several corticolimbic brain structures after retrieval of consolidated fear memories $[39,40]$. Nevertheless, lesion studies in contextual 
fear memory did not show any disruption of contextual fear retrieval following inactivation of the dorsal hippocampal CA1 or CA3 subregions during a contextual memory test [37].

The molecular events underlying learning and memory have increasingly become an area of intense interest. The present findings provide evidence for spatial probe test-induced changes in pCREB and Egr1 in the hippocampus of aged mice. The findings of this and other studies [41] support the hypothesis that expression of pCREB and Egr1 in the hippocampus is a crucial step for spatial memory reactivation. Hippocampus-dependent memory impairments are prevalent in both aged humans and rodents [42]. Based on these findings, we are currently carrying out further experiments to examine whether retrieval is impaired when pCREB and Egr1 expression is pharmacologically abolished, and to show whether there is a causal relationship between pCREB and Egr1 expression and retrieval of spatial memory.

\section{Acknowledgement}

We thank Dr. Yang Wang for technical assistance and Dr. Hua Yang for her help in animal breeding and care. We received funding from the Shanghai Natural Science Foundation (10ZR1406500).

\section{Disclosure Statement}

The authors have no conflicts of interest to disclose.

\section{References}

- 1 Kandel ER: Genes, synapses, and long-term memory. J Cell Physiol 1997;173:124-125.

- 2 Dash PK, Hochner B, Kandel ER: Injection of the cAMP-responsive element into the nucleus of Aplysia sensory neurons blocks long-term facilitation. Nature 1990;345:718-721.

- 3 Yin JC, Wallach JS, Del Vecchio M, Wilder EL, Zhou H, Quinn WG, et al: Induction of a dominant negative CREB transgene specifically blocks long-term memory in Drosophila. Cell 1994;79:49-58.

- 4 Kogan JH, Frankland PW, Blendy JA, Coblentz J, Marowitz Z, Schutz G, et al: Spaced training induces normal long-term memory in CREB mutant mice. Curr Biol 1997; 7:1-11.

- 5 Guzowski JF, McGaugh JL: Antisense oligodeoxynucleotide-mediated disruption of hippocampal cAMP response element binding protein levels impairs consolidation of memory for water maze training. Proc Natl Acad Sci USA 1997;94:2693-2698.

- 6 Rosenegger D, Parvez K, Lukowiak K: Enhancing memory formation by altering protein phosphorylation balance. Neurobiol Learn Mem 2008;90:544-552.

- 7 Brightwell JJ, Smith CA, Neve RL, Colombo PJ: Long-term memory for place learning is facilitated by expression of cAMP response element-binding protein in the dorsal hippocampus. Learn Mem 2007;14:195-199.

$\checkmark 8$ Trifilieff P, Herry C, Vanhoutte P, Caboche J, Desmedt A, Riedel G, et al: Foreground contextual fear memory consolidation requires two independent phases of hippocampal ERK/CREB activation. Learn Mem 2006;13:349-358.

- 9 Martel G, Millard A, Jaffard R, Guillou JL: Stimulation of hippocampal adenylyl cyclase activity dissociates memory consolidation processes for response and place learning. Learn Mem 2006;13:342-348.

10 Moncada D, Viola H: Phosphorylation state of CREB in the rat hippocampus: a molecular switch between spatial novelty and spatial familiarity? Neurobiol Learn Mem 2006;86:9-18.

11 Porte Y, Buhot MC, Mons N: Alteration of CREB phosphorylation and spatial memory deficits in aged 129T2/ Sv mice. Neurobiol Aging 2008;29:1533-1546.

12 Pittenger C, Huang YY, Paletzki RF, Bourtchouladze R, Scanlin H, Vronskaya S, et al: Reversible inhibition of CREB/ATF transcription factors in region CA1 of the dorsal hippocampus disrupts hippocampus-dependent spatial memory. Neuron 2002;34:447-462.

13 Florian C, Mons N, Roullet P: CREB antisense oligodeoxynucleotide administration into the dorsal hippocampal CA3 region impairs long- but not short-term spatial memory in mice. Learn Mem 2006;13:465-472.

14 Xu J, Rong S, Xie B, Sun Z, Deng Q, Wu H, et al: Memory impairment in cognitively impaired aged rats associated with decreased hippocampal CREB phosphorylation: reversal by procyanidins extracted from the lotus seedpod. J Gerontol A Biol Sci Med Sci 2010;65:933-940. 
Alberini CM: Transcription factors in long-term memory and synaptic plasticity. Physiol Rev 2009;89:121-145. system. Neuron 1990;4:477-485.

17 Kida S, Josselyn SA, Pena de Ortiz S, Kogan JH, Chevere I, Masushige S, et al: CREB required for the stability of new and reactivated fear memories. Nat Neurosci 2002;5:348-355.

18 Sung JY, Goo JS, Lee DE, Jin DQ, Bizon JL, Gallagher M, et al: Learning strategy selection in the water maze and hippocampal CREB phosphorylation differ in two inbred strains of mice. Learn Mem 2008;15:183-188.

19 Morris R: Developments of a water-maze procedure for studying spatial learning in the rat. J Neurosci Methods 1984;11:47-60.

20 Beiko J, Candusso L, Cain DP: The effect of nonspatial water maze pretraining in rats subjected to serotonin depletion and muscarinic receptor antagonism: a detailed behavioural assessment of spatial performance. Behav Brain Res 1997;88:201-211.

21 Bisler S, Schleicher A, Gass P, Stehle JH, Zilles K, Staiger JF: Expression of c-Fos, ICER, Krox-24 and JunB in the whisker-to-barrel pathway of rats: time course of induction upon whisker stimulation by tactile exploration of an enriched environment. J Chem Neuroanat 2002;23:187-198.

-22 Zangenehpour S, Chaudhuri A: Differential induction and decay curves of c-fos and zif268 revealed through dual activity maps. Brain Res Mol Brain Res 2002;109:221-225.

23 Porte Y, Buhot MC, Mons NE: Spatial memory in the Morris water maze and activation of cyclic AMP response element-binding (CREB) protein within the mouse hippocampus. Learn Mem 2008;15:885-894.

24 Mizuno M, Yamada K, Maekawa N, Saito K, Seishima M, Nabeshima T: CREB phosphorylation as a molecular marker of memory processing in the hippocampus for spatial learning. Behav Brain Res 2002;133:135-141.

-25 Colombo PJ, Brightwell JJ, Countryman RA: Cognitive strategy-specific increases in phosphorylated cAMP response element-binding protein and c-Fos in the hippocampus and dorsal striatum. J Neurosci 2003;23: 3547-3554.

-26 Yin JC, Del Vecchio M, Zhou H, Tully T: CREB as a memory modulator: induced expression of a dCREB2 activator isoform enhances long-term memory in Drosophila. Cell 1995;81:107-115.

27 Davis S, Vanhoutte P, Pages C, Caboche J, Laroche S: The MAPK/ERK cascade targets both Elk-1 and cAMP response element-binding protein to control long-term potentiation-dependent gene expression in the dentate gyrus in vivo. J Neurosci 2000;20:4563-4572.

-28 Zhang JJ, Okutani F, Inoue S, Kaba H: Activation of the mitogen-activated protein kinase/extracellular signalregulated kinase signaling pathway leading to cyclic AMP response element-binding protein phosphorylation is required for the long-term facilitation process of aversive olfactory learning in young rats. Neuroscience 2003;121:9-16.

29 Brightwell JJ, Smith CA, Countryman RA, Neve RL, Colombo PJ: Hippocampal overexpression of mutant creb blocks long-term, but not short-term memory for a socially transmitted food preference. Learn Mem 2005;12: 12-17.

-30 Costa-Mattioli M, Gobert D, Harding H, Herdy B, Azzi M, Bruno M, et al: Translational control of hippocampal synaptic plasticity and memory by the eIF2alpha kinase GCN2. Nature 2005;436:1166-1173.

- 31 Huang YY, Kandel ER: Modulation of both the early and the late phase of mossy fiber LTP by the activation of beta-adrenergic receptors. Neuron 1996;16:611-617.

-32 Bernabeu R, Bevilaqua L, Ardenghi P, Bromberg E, Schmitz P, Bianchin M, et al: Involvement of hippocampal cAMP/cAMP-dependent protein kinase signaling pathways in a late memory consolidation phase of aversively motivated learning in rats. Proc Natl Acad Sci USA 1997;94:7041-7046.

33 Jones MW, Errington ML, French PJ, Fine A, Bliss TV, Garel S, et al: A requirement for the immediate early gene Zif268 in the expression of late LTP and long-term memories. Nat Neurosci 2001;4:289-296.

-34 Nakazawa K, Quirk MC, Chitwood RA, Watanabe M, Yeckel MF, Sun LD, et al: Requirement for hippocampal CA3 NMDA receptors in associative memory recall. Science 2002;297:211-218.

- 35 Rudy JW, O’Reilly RC: Conjunctive representations, the hippocampus, and contextual fear conditioning. Cogn Affect Behav Neurosci 2001;1:66-82.

-36 Schlingensiepen KH, Luno K, Brysch W: High basal expression of the zif/268 immediate early gene in cortical layers IV and VI, in CA1 and in the corpus striatum - an in situ hybridization study. Neurosci Lett 1991;122:67-70.

- 37 Daumas S, Halley H, Frances B, Lassalle JM: Encoding, consolidation, and retrieval of contextual memory: differential involvement of dorsal CA3 and CA1 hippocampal subregions. Learn Mem 2005;12:375-382.

-38 Nakazawa K, Sun LD, Quirk MC, Rondi-Reig L, Wilson MA, Tonegawa S: Hippocampal CA3 NMDA receptors are crucial for memory acquisition of one-time experience. Neuron 2003;38:305-315.

39 Hall J, Thomas KL, Everitt BJ: Cellular imaging of zif268 expression in the hippocampus and amygdala during contextual and cued fear memory retrieval: selective activation of hippocampal CA1 neurons during the recall of contextual memories. J Neurosci 2001;21:2186-2193.

40 Thomas KL, Hall J, Everitt BJ: Cellular imaging with zif268 expression in the rat nucleus accumbens and frontal cortex further dissociates the neural pathways activated following the retrieval of contextual and cued fear memory. Eur J Neurosci 2002;16:1789-1796.

-41 Poirier GL, Amin E, Aggleton JP: Qualitatively different hippocampal subfield engagement emerges with mastery of a spatial memory task by rats. J Neurosci 2008;28:1034-1045.

42 Driscoll I, Sutherland RJ: The aging hippocampus: navigating between rat and human experiments. Rev Neurosci 2005;16:87-121. 